\title{
Necrológio
}

\section{Rosária de Rosa Barbosa}

\author{
$31.01 .1947-22.08 .2005$
}

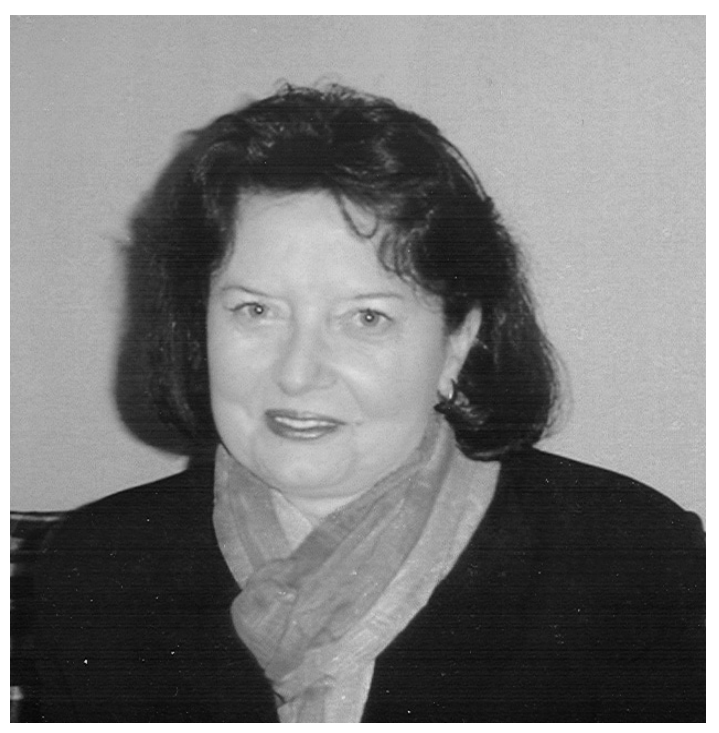

ROSÁRIA DE ROSA BARBOSA nasceu em Santa Maria Capua Vetere, região da Campania, Itália, no dia 31 de janeiro de 1947. Em 1955, em companhia dos pais Salvatore e Raffaela De Rosa, chegou a Porto Alegre, vinda de Nápolis, região de Caseserta. Fez seu curso secundário no Colégio Batista e posteriormente ingressou no Instituto de Biociências da Universidade Federal do Rio Grande do Sul, onde licenciou-se em História Natural em 1971. Na Pontifícia Universidade Católica do Rio Grande do Sul obteve, em 1983, título de mestre em Zoologia, com a dissertação "Reavaliação da fauna espongológica continental do Rio Grande do Sul frente a novas coletas".

Iniciou sua carreira científica em 1970, como estagiária voluntária no Setor de Porifera do então Museu Rio-grandense de Ciências Naturais, sob orientação da Dra. Cecília Volkmer Ribeiro e, a seguir, com estágios de Iniciação Técnica Remunerada até 1971, através de auxílios concedidos pela FAPERGS à orientadora. Com a mesma orientação foi, a seguir, bolsista de Iniciação Científica do CNPq. Seguiu no Setor de Porifera como bolsista de Aperfeiçoamento do CNPq, nos exercícios de 1973 e 1974, bolsista Pesquisador-Assistente do CNPq de 1975 a 1977 e, como Bolsista-Pesquisador, de 1978 a 1992. Estagiou no Museu Nacional de História Natural de Montevideo, Uruguai e no Instituto Nacional de Limnologia, Santa Fé, Argentina. Em maio de 1995, no Instituto de Zoologia da Universidade de Gênova, Itália, juntamente com o Dr. Michele Sarà, realizou estudo sobre esponjas marinhas do litoral sul-brasileiro. Foi beneficiária de auxílios à pesquisa que propôs à FAPERGS e ao CNPq.

Ingressou, em 1975, no quadro funcional da Fundação Zoobotânica do Rio Grande do Sul, como professora cedida do Ensino Médio de Ciências Físicas e Biológicas, exercendo sempre sua atividade na equipe de Porifera dedicada aos estudos da fauna de esponjas continentais. Em outubro de 1988 exonerou-se do magistério estadual, ingressando no quadro permanente de pessoal da Fundação Zoobotânica do Rio Grande do Sul, como Técnico Superior Pesquisador, lotada no Setor Porifera, Núcleo de Invertebrados Inferiores do Museu de Ciências Naturais. Exerceu a chefia desse núcleo de 1990 a 2004. Orientou 11 bolsistas de Iniciação Científica, do CNPq e da FAPERGS, além de 11 acadêmicas dos cursos de Biologia, em estágios regulares de Iniciação Técnica.

Foi curadora da Coleção de Cnidários desde 1990, havendo no entanto contribuído para o incremento da coleção de esponjas continentais do Estado, por sua atividade em inúmeros projetos, tanto gerais do Museu - e nos quais a equipe de Porifera se inseriu -, quanto projetos específicos dessa equipe. Atuou como membro da comissão redatorial do Periódico Iheringia, Série Zoologia, de 1986 a 1990.

Participou de vários congressos, simpósios, cursos e seminários, além de atividades museológicas, tanto buscando conhecimento, quanto contribuindo para a divulgação das pesquisas do Setor de Porifera e do Museu. 
Foi membro de diversas associações profissionais e científicas, havendo atuado como tesoureira do Conselho Regional de Museologia (1996/1998 e 2004/ 2005).

Rosária De Rosa-Barbosa foi casada com Humberto Barbosa e deixou as filhas Lisiane e Luciana e o neto Bruno.

Seu falecimento prematuro cria uma lacuna no já reduzido contingente de pesquisadores brasileiros dedicados ao estudo das esponjas e um vazio maior ainda no coração dos colegas e amigos, para quem sempre tinha uma palavra de carinho e tranqüilidade.

\section{Cecília Volkmer Ribeiro}

\section{TRABALHOS PUBLICADOS}

De Rosa-Barbosa, R. colab. 1976. In: Fundação Zoobotânica do Rio Grande do Sul/MCN. Preceituação ecológica para a preservação de recursos naturais na região da Grande Porto Alegre. FZB/Sulina. 153p. (Publicações Avulsas FZB, 1)

De Rosa-Barbosa, R. 1979. Redescrição do material tipo de Ephydatia facunda Weltner, 1895 (Porifera-Spongillidae). Iheringia, Série Zoologia, (54):27-34.

De Rosa-Barbosa, R. 1980. Redescrição de Drulia cristata (Weltner, 1895) e identificação do conteúdo espicular associado (Porifera - Spongillidae). Iheringia, Série Zoologia, (56): 87-94

De RosA-BARbosA, R. 1984. Reavaliação da fauna espongológica continental do Rio Grande do Sul frente a novas coletas. Iheringia, Série Zoologia, (64):127-148

De Rosa-Barbosa, R. 1986. Corvospongilla volkmeri sp.n. e registro de Corvospongilla seckti Bonetto \& Ezcurra de Drago,1966 no Brasil (Porifera, Spongillidae). Iheringia, Série Zoologia, (67):109-122.

De Rosa-Barbosa, R. 1995. Primeiro registro de Myrmekioderma styx Laubenfels, 1953 (Porifera - Demospongiae) no Atlântico Sudoeste com novos aportes para a caracterização da espécie. Biociências 3(2):119-128.

De Rosa-Barbosa, R. \& Mothes de Moraes, B. 1978. As esponjas. Natureza em Revista 4:14-17.

Sarà, M. \& De Rosa-Barbosa, R. 1995. A new species of Halicometes from the southern Brazilian coast (Porifera Tethyidae). Bollettino di Zoologia 62:167-171.

Tavares, M. C. M.; Volkmer-Ribeiro, C. \& De Rosa-Barbosa, R. 2003. Primeiro registro de Corvoheteromeyenia australis (Bonetto \& Ezcurra de Drago) para o Brasil com chave taxonômica para os poríferos do Parque Estadual Delta do Jacuí, Rio Grande do Sul, Brasil. Revista Brasileira de Zoologia 20(2):169-182.

Volkmer-Ribeiro, C. \& De Rosa-Barbosa, R. 1972. On Acalle recurvata (Bowerbank, 1863) and an associated fauna of other freshwater sponges. Revista Brasileira de Biologia 32(3):303-317

Volkmer-Ribeiro, C.; De Rosa-Barbosa, R.; Mothes de Moraes, B. \& Grosser, K. M. 1973. Relatório sobre a segunda pesquisa oceanográfica e pesqueira do Atlântico Sul entre Torres e Maldonado (Lat. $29^{\circ} \mathrm{S}-35^{\circ} \mathrm{S}$ ). Programa Rio Grande do Sul
III. Nota Preliminar sobre Porifera. Publicação Especial do Instituto Oceanográfico 3(1):233-237.

Volkmer-Ribeiro, C. \& DE Rosa-Barbosa, R. 1974. A freshwater sponge mollusk association in Amazonia waters. Amazoniana 5(2):285-291.

Volkmer-Ribeiro, C.; Grosser, K. M.; De Rosa-Barbosa, R. \& Pauls, S. M. 1975. Primeiro relato da ocorrência de espongilídeos (Porifera) na bacia do Guaíba, Estado do Rio Grande do Sul. Iheringia, Série Zoologia, (46):33-49.

Volkmer-Ribeiro, C. \& De Rosa-Barbosa, R. 1978. A new genus and species of Neotropical freshwater sponges. Iheringia, Série Zoologia, (52):103-107.

Volkmer-Ribeiro, C. \& De Rosa-Barbosa, R. 1978. Neotropical freshwater sponges of the family Potamolepidae Brien, 1967. In: Levi, C. \& Boury-Esnault, N. Biologia des Spongiaires, Sponge Biology. Paris, Centre National de la Recherche Scientifique. p. 503-511, il. (Colloques Internationaux du Centre National de Recherche Scientifique, 291).

Volkmer-Ribeiro, C.; De Rosa-Barbosa, R. \& Mansur, M. C. D. 1981. Fauna espongológica e malacológica bêntica da Lagoa Negra, Parque Estadual de Itapuã, Rio Grande do Sul. Iheringia, Série Zoologia, (59):13-24.

Volkmer-Ribeiro, C.; De Rosa-Barbosa, R. \& Mello, H. F. 1983. The unexpected ocurrence of Drulia browni (Bowerbank, 1863) (Porifera - Spongillidae) in an oxbow lake at the extreme south of Brazil. Iheringia, Série Zoologia, (63):3-10.

Volkmer-Ribeiro, C.; Mothes-de-Moraes, B.; De Rosa-Barbosa, R.; Mansur, M. C. D. \& Veitenheimer-Mendes, I. L. 1984. Um estudo do bentos em raízes de Eichornia azurea (Sw.) Kunth, do curso inferior de um rio subtropical Sul-Americano e de um pequeno afluente. Revista Brasileira de Biologia 44(2):125-132.

Volkmer-Ribeiro, C.; De Rosa-Barbosa, R. 1985. Redescription of the freshwater sponges Trochospongilla repens (Hinde, 1888) and Trochospongilla amazonica (Weltner, 1895) with an account of the South American species of Trochospongilla (Porifera, Spongillidae). Iheringia, Série Zoologia, (65):77-93.

Volkmer-Ribeiro, C.; De Rosa-Barbosa, R. \& Marques-Tavares, M C. 1988. Anheteromeyenia sheilae sp. n. e outras esponjas dulciaquícolas da região costeira do Rio Grande do Sul. Iheringia, Série Zoologia, (68):83-98.

Volkmer-Ribeiro, C.; Gastal, H. A.O.; Silva, M. C. P.; Lanzer-deSouza, M. E.; Fabián, M. H.; Braun, P. C.; De Rosa-Barbosa, R \& LEMA, T. DE. 1990. Levantamento de invertebrados aquáticos, anfíbios, répteis e morcegos, microbacia piloto do Arroio Umbú. In: Estudo básico da microbacia do Arroio Umbú, Victor Graeff - RS. Porto Alegre, IPRNR. p.49-57. (Publicação IPRNR, 23).

Volkmer-Ribeiro, C.; De Rosa-Barbosa, R. 1992. Esponjas de água doce. In: Guia ilustrado de flora e fauna para o Parque Copesul de Proteção Ambiental. Porto Alegre, Copesul. 209p., il.

Volkmer-Ribeiro, C.; De Rosa-Barbosa, R.; Tavares, M. Da C. M. \& Silva, M. M. DA. 2002. Sinaleiras no Delta, esponjas. Natureza em Revista 13:28-35

Volkmer-Ribeiro, C.; Guadagnin, D. L.; De Rosa-Barbosa, R.; Silva, M. M.; DrügG-Hahn, S.; Lopes-Pitoni, V. L.; Gastal, H. A. DE O.; Barros, M. P. DE \& Demaman, L. V. 2004. A Polyethylenetherephthalate (PET) device for sampling freshwater benthic macroinvertebrates. Brazilian Journal of Biology 64(3):1-8. 